\title{
Morfologia, características da carcaça e componentes do peso vivo em borregos Corriedale e Ideal
}

\author{
Morphology, carcass characteristics and liveweight \\ components in Corriedale and Polwarth wethers
}

\author{
Gilson de Mendonça ${ }^{1}$ José Carlos Osório ${ }^{1,2}$ Nelson Manzoni Oliveira ${ }^{2,3}$ \\ Maria Tereza Osório ${ }^{1,2}$ Roger Esteves ${ }^{4}$ Mabel Mascaranhas Wiengard ${ }^{1}$
}

\section{RESUMO}

O objetivo deste trabalho foi comparar às raças Corriedale e Ideal quanto as suas características morfológicas e da carcaça, bem como avaliar a variação do peso vivo e de seus componentes. Foram utilizados 40 borregos (castrados aos 30 dias de idade) das raças Ideal $(n=19)$ e Corriedale $(n$ = 21), criados em condições extensivas, com alimentação exclusiva em pasto nativo, desmamados aos 70 dias e abatidos com 1 ano de idade. A fase experimental de campo foi realizada na EMBRAPA - CPPSUL de Bagé/RS, e a fase laboratorial na UFPel - FAEM - Departamento de Zootecnia, Pelotas/RS. A raça Corriedale apresentou valores maiores $(P<0,05)$ para conformação, condição corporal, comprimento corporal (todas in vivo) e comprimento da perna in vivo e na carcaça. Para as demais características morfológicas, principalmente aquelas avaliadas na carcaça, não foram encontradas diferenças $(P>0,05)$. A raça Corriedale apresentou maior peso vivo ao abate e maior peso de carcaça quente, observando-se na raça Ideal as menores perdas por resfriamento, tanto em gramas quanto em percentagem $(P<0,05)$. Não houve efeito de raça no peso de carcaça fria e nos rendimentos verdadeiro e comercial $(P>0,05)$. Os borregos Corriedale apresentaram maiores pesos de patas e pulmóes com traquéia e menor peso de gordura interna. Em valores relativos (\%), os borregos da raça Ideal apresentaram uma proporção maior de fígado e de gordura interna. Concluiu-se que diferenças morfológicas entre as raças estudadas foram observadas somente in vivo. Já os componentes do peso vivo, apresentaram uma variação, quantitativa e qualitativa, em ambas as raças.

Palavras-chave: componentes do peso vivo, carcaça, carne, borregos, raças.

\section{ABSTRACT}

The objective of this study was to compare Corriedale and Polwarth breeds as to morphologic and carcass characteristics, as well as, to evaluate liveweight and liveweight components variation. Forty wethers (castrated at 30 days of age), were used (19 Polwarth and 21 Corriedale), weaned at 70 days, and slaughtered with one year of age. Animals were raised under extensive native pasture conditions at Embrapa Pecuária Sul - CPPSUL, Bagé, RS. Laboratory measurements were carried out at the Animal Science Department of Universidade Federal de Pelotas, in Pelotas, RS. Corriedale breed showed higher values $(P<0.05)$ for in vivo body conformation, body condition, body length and leg length. Other morphological carcass characteristics did not show significant differences $(P>0.05)$. Corriedale breed presented higher slaughtering weight and higher hot carcass weight. Polwarth showed lower cooling losses, both in weight and percentage $(P<0.05)$. Cold carcass weight and yield were not different between breeds $(P>0.05)$. Corriedale wethers presented higher weight for feet and lungs and lower weight for internal fat. In relative values, Polwarth wethers showed higher

${ }^{1}$ Médico Veterinário, Professor, Universidade Federal de Pelotas (UFPel), UFPEL/FAEM/DZ, Campus Universitário s/n, CP 354, 96010-900, Pelotas, RS. Autor para correspondência

${ }^{2}$ Bolsista $\mathrm{CNPq}$

${ }^{3}$ Médico Veterinário, Doutor, pesquisador da EMBRAPA-CPPSUL, Bagé, RS

${ }^{4}$ Aluno do curso de Agronomia, UFPEL/FAEM/DZ, Campus Universitário s/n, CP 354, 96010-900, Pelotas, RS. 
proportion of liver and internal fat. It is concluded that morphological differences are observed only in live animals. Liveweight components showed quantitative and qualitative differences for both breeds.

Key words: liveweight components, carcass, meat, wethers, breeds.

\section{INTRODUÇÃO}

O rebanho ovino existente no estado do Rio Grande do Sul, embora tenha sofrido significativa redução nos últimos anos, oscila em torno de 4,8 milhões de cabeças (IBGE, 2001) e é uma atividade econômica importante para a região; sendo ultimamente destaque a produção de carne ovina. As raças Corriedale e Ideal são as principais criadas no Rio Grande do Sul, entretanto, poucos são os estudos comparativos sobre a produção de carne, avaliação da morfologia e dos componentes do peso vivo, o que limita a informação sobre o potencial produtivo de tão importantes genótipos.

A carcaça é o componente do peso vivo de maior valor comercial, entretanto, os demais (pele, cabeça, patas, vísceras verdes e vermelhas), também chamados componentes não constituintes da carcaça, freqüentemente representam uma parte ponderal mais importante que a carne (FRAYSSE \& DARRE, 1990). Para uma valorização comercial justa do animal como um todo, deveria levar-se em consideração os componentes não constituintes da carcaça, uma vez que este poderia tornar-se uma fonte alternativa de alimento, estimulando o criador a zelar pelas condições sanitárias de seus animais (PRADAL, 1989; OSÓRIO et al., 1995; OSÓRIO et al., 1996a; OSÓRIO et al., 1996b). OSÓRIO et al. (1996a), trabalhando com cinco genótipos, verificaram que entre as raças Corriedale, Ideal e Merino não existiam diferenças nos componentes do peso vivo que justificassem uma valorização diferenciada, apresentando os animais um crescimento ponderal, do desmame (70 dias) ao abate (225 dias), semelhante.

Uma das principais fontes de variação na quantidade e qualidade das carcaças, assim como nos componentes não constituintes da carcaça, é o genótipo, ao lado de outros fatores tais como estado sanitário, idade e sexo (COLOMER \& ESPEJO DIAZ, 1971; OSÓRIO et al., 1991; SIERRA et al., 1993; OSÓRIO et al., 1995; OSÓRIO et al., 1996c; OSÓRIO et al., 2000). De acordo com a aptidão da raça (carne ou lã), podem encontrar-se diferentes valores para a carcaça (OLIVEIRA et al., 1998), e a influência de alguns componentes no peso vivo diminui à medida que a raça especializa-se para a produção de carne (OSÓRIO et al., 1996c).
COSTA et al. (1999) verificaram que não houve diferenças nas características morfológicas, in vivo e na carcaça, entre as raças Corriedale e Ideal, bem como em determinadas características de interesse comercial, em cordeiros com 222 dias de idade. Entretanto, a idade é um fator importante e determinante da composição corporal e tecidual, tanto na raça Corriedale como na Ideal (OSÓRIO et al., 2000), mostrando ser necessária a realização de estudos em diferentes idades.

O objetivo deste trabalho foi comparar borregos com 1 ano de idade, das raças Ideal e Corriedale, quanto à morfologia in vivo e na carcaça, características de interesse comercial e variação do peso vivo e de seus componentes.

\section{MATERIALEMÉTODOS}

O experimento foi realizado na Embrapa Pecuária Sul (CPPSUL) de Bagé - RS, com a participação (fase laboratorial) do Departamento de Zootecnia da Faculdade de Agronomia Eliseu Maciel da Universidade Federal de Pelotas, nos anos de 1999/ 2000. Utilizaram-se 40 borregos castrados aos 30 dias de idade, das raças Ideal $(n=19)$ e Corriedale $(n=21)$, nascidos entre 20/09/1999 e 30/10/1999. Foram criados em campo nativo, com predominância de capim anoni (Eragrostis plana Nees), tendo sido desmamados aos 70 dias e abatidos com idade média de 1 ano, nos dias 20 e 28 de novembro de 2000.

Antes do sacrifício e após jejum de 14 horas, foram feitas as seguintes medições: peso vivo ao abate, conformação in vivo, condição corporal, comprimento corporal, perímetro torácico, altura, comprimento da perna e compacidade do animal vivo. Imediatamente após o abate, foram tomados os pesos da carcaça quente, cabeça, patas, pele, coração, pulmões com traquéia, baço, fígado, gordura interna, diafragma e vísceras verdes cheias. Determinou-se, subjetivamente, o estado de engorduramento e a conformação da carcaça. Foi calculada a percentagem de cada componente não constituinte da carcaça em relação ao peso vivo de abate.

Após resfriamento em câmara fria com ar forçado a $0^{\circ} \mathrm{C}$, durante 17 horas, foram feitas as seguintes mensurações: peso da carcaça fria, comprimento interno da carcaça, comprimento da perna, largura da perna, profundidade da perna, gordura pélvica e renal, textura, cor, marmoreio e peso dos rins. Foi ainda avaliada a espessura da gordura de cobertura, rendimentos verdadeiro e comercial da carcaça, bem como sua compacidade e as perdas por resfriamento. A metodologia utilizada para determinação das variáveis acima citadas foi a descrita por OSÓRIO et al. (1998). 
Através da análise de variância dos dados verificou-se o efeito do genótipo sobre as características morfológicas e de interesse comercial, assim como sobre o peso vivo ao abate e seus componentes. A análise foi feita através do PROC GLM (General Linear Models), do programa Statistical Analysis System (SAS, 1982).

\section{RESULTADOS E DISCUSSÃO}

A análise da variância demonstrou ter havido diferença significativa $(\mathrm{P}<0,05)$ entre os genótipos para: conformação, condição corporal, comprimento corporal, todas medidas in vivo e para comprimento da perna, in vivo e na carcaça, sendo que a raça Corriedale apresentou os maiores valores (Tabela 1). As médias encontradas no presente estudo foram superiores às verificadas por OSÓRIO et al. (1996c), em animais mais jovens das mesmas raças, especialmente no comprimento corporal e no comprimento da perna in vivo e na carcaça, salientando que, de maneira inversa a este trabalho, os referidos autores não encontraram efeito de genótipo. De acordo com SAÑUDO \& SIERRA (1986), a base genética, aliada ao estado de engorduramento, é uma das principais responsáveis pela variação na conformação e condição corporal, sendo que a gordura foi a responsável pela diferença na conformação e condição corporal entre as raças (Tabela 1).

Para as demais características morfológicas, não se observou diferenças entre as raças $(\mathrm{P}>0,05)$. Os resultados encontrados, especialmente para compacidade (in vivo e da carcaça) e conformação da carcaça, demonstraram que as diferenças morfológicas podem ser detectáveis no animal vivo, não se observando a mesma tendência quando avaliadas na carcaça. Estes resultados são semelhantes aos encontrados por OSÓRIO et al. (1996c) e COSTA et al. (1999), indicando a importância da avaliação da carcaça.

Com relação às características da carcaça, a raça Corriedale apresentou maior peso vivo ao abate e maior peso de carcaça quente $(\mathrm{P}<0,05)$, entretanto, a proporção de carcaça em relação ao peso vivo, componente mais valorizado, não diferiu $(\mathrm{P}>0,05)$. Por outro lado, a raça Ideal apresentou menores perdas por resfriamento, tanto em gramas quanto em percentagem, o que pode ter sido responsável pela ausência de diferenças no peso de carcaça
Tabela 1 - Médias e erros padrão da morfologia in vivo e na carcaça, segundo a raça.

\begin{tabular}{lcc}
\hline Parâmetros & Ideal & Corriedale \\
\hline Morfologia in vivo & & \\
& & \\
Conformação (índice 1 a 5) & $2,18 \pm 0,07 \mathrm{a}$ & $2,47 \pm 0,07 \mathrm{~b}$ \\
Condição corporal (índice 1 a 5) & $2,36 \pm 0,08$ a & $2,61 \pm 0,08 \mathrm{~b}$ \\
Comprimento corporal (cm) & $57,63 \pm 0,82$ a & $59,90 \pm 0,78 \mathrm{~b}$ \\
Perímetro torácico (cm) & $77,73 \pm 1,00$ a & $77,95 \pm 0,95 \mathrm{a}$ \\
Altura (cm) & $57,42 \pm 0,73$ a & $59,19 \pm 0,69 \mathrm{a}$ \\
Comprimento da perna (cm) & $51,57 \pm 0,56$ a & $53,33 \pm 0,54 \mathrm{~b}$ \\
Compacidade corporal (kg/cm) & $0,59 \pm 0,01 \mathrm{a}$ & $0,62 \pm 0,01 \mathrm{a}$ \\
Morfologia na carcaça & & \\
& & \\
Comprimento interno da carcaça (cm) & $54,96 \pm 0,93$ a & $56,25 \pm 0,88 \mathrm{a}$ \\
Comprimento da perna (cm) & $38,02 \pm 0,35$ a & $39,95 \pm 0,34 \mathrm{~b}$ \\
Largura da perna (cm) & $8,01 \pm 0,20 \mathrm{a}$ & $8,36 \pm 0,19 \mathrm{a}$ \\
Profundidade da perna (cm) & $13,37 \pm 0,61$ a & $14,05 \pm 0,58 \mathrm{a}$ \\
Conformação (índice 1 a 5) & $2,10 \pm 0,11 \mathrm{a}$ & $2,07 \pm 0,10 \mathrm{a}$ \\
Compacidade da carcaça (kg/cm) & $0,24 \pm 0,09$ a & $0,26 \pm 0,09$ a \\
\hline
\end{tabular}

Médias na mesma linha, seguidas de letras diferentes, diferem entre si $(\mathrm{P}<0,05)$.

fria e nos rendimentos verdadeiro e comercial (Tabela 2). OLIVEIRA et al. (2000), trabalhando com cordeiros cruza Border Leicester x Corriedale e Border Leicester x Ideal, verificaram menores perdas por resfriamento no cruzamento com Ideal, atribuindo os resultados ao efeito genético do cruzamento e, possivelmente, à distribuição da gordura de cobertura. OSÓRIO et al. (1996c) e COSTA et al. (1999), de maneira semelhante a este trabalho e apenas com o diferencial de haverem trabalhado com cordeiros, encontraram similaridade entre as raças Corriedale e Ideal para peso de carcaça fria e rendimento de carcaça, mostrando que as duas raças podem fazer parte de uma mesma categoria comercial.

Tabela 2 - Médias e erros padrão das características comerciais da carcaça, segundo a raça.

\begin{tabular}{lcc}
\hline Parâmetros & Ideal & Corriedale \\
\hline & & \\
Peso de carcaça quente (kg) & $13,85 \pm 0,40 \mathrm{a}$ & $15,32 \pm 0,38 \mathrm{~b}$ \\
Peso de carcaça fria (kg) & $13,33 \pm 0,42 \mathrm{a}$ & $14,43 \pm 0,40 \mathrm{a}$ \\
Estado de engorduramento (índice 1 a 5) & $2,02 \pm 0,11 \mathrm{a}$ & $1,90 \pm 0,10 \mathrm{a}$ \\
Gordura pélvica e renal (kg) & $0,20 \pm 0,01 \mathrm{a}$ & $0,16 \pm 0,01 \mathrm{a}$ \\
Rendimento verdadeiro (\%) & $40,50 \pm 0,96 \mathrm{a}$ & $40,80 \pm 0,92 \mathrm{a}$ \\
Rendimento comercial (\%) & $38,52 \pm 0,90 \mathrm{a}$ & $38,39 \pm 0,85 \mathrm{a}$ \\
Perda por resfriamento (kg) & $0,51 \pm 0,08 \mathrm{a}$ & $0,89 \pm 0,08 \mathrm{~b}$ \\
Perda por resfriamento (\%) & $3,77 \pm 0,64 \mathrm{a}$ & $5,94 \pm 0,61 \mathrm{~b}$ \\
Espessura da gordura de cobertura (cm) & $0,02 \pm 0,00 \mathrm{a}$ & $0,02 \pm 0,00 \mathrm{a}$ \\
Textura (índice 1 a 5) & $2,47 \pm 0,09 \mathrm{a}$ & $2,59 \pm 0,09 \mathrm{a}$ \\
Cor (índice 1 a 5) & $2,50 \pm 0,10 \mathrm{a}$ & $2,76 \pm 0,09 \mathrm{a}$ \\
Marmoreio (índice 1 a 5) & $1,39 \pm 0,09 \mathrm{a}$ & $1,45 \pm 0,08 \mathrm{a}$ \\
\hline
\end{tabular}

Médias na mesma linha, seguidas de letras diferentes, diferem entre si $(\mathrm{P}<0,05)$. 
Tabela 3 - Médias e erro padrão, em kg e em \%, dos componentes do peso vivo, segundo a raça.

\begin{tabular}{lcc}
\hline Parâmetros & Ideal & Corriedale \\
\hline Em kg & & \\
& & \\
Peso vivo ao abate (PV) & $34,61 \pm 0,49 \mathrm{a}$ & $37,50 \pm 0,90 \mathrm{~b}$ \\
Peso de carcaça quente & $13,85 \pm 0,40 \mathrm{a}$ & $15,32 \pm 0,38 \mathrm{~b}$ \\
Cabeça & $1,36 \pm 0,04 \mathrm{a}$ & $1,44 \pm 0,02 \mathrm{a}$ \\
Patas & $0,73 \pm 0,02 \mathrm{a}$ & $0,81 \pm 0,02 \mathrm{~b}$ \\
Pele & $6,78 \pm 0,21 \mathrm{a}$ & $7,23 \pm 0,20 \mathrm{a}$ \\
Coração & $0,17 \pm 0,01 \mathrm{a}$ & $0,18 \pm 0,01 \mathrm{a}$ \\
Pulmões + traquéia & $0,61 \pm 0,02 \mathrm{a}$ & $0,68 \pm 0,02 \mathrm{~b}$ \\
Baço & $0,09 \pm 0,01 \mathrm{a}$ & $0,09 \pm 0,01 \mathrm{a}$ \\
Fígado & $0,58 \pm 0,02 \mathrm{a}$ & $0,58 \pm 0,01 \mathrm{a}$ \\
Gordura interna & $0,27 \pm 0,03 \mathrm{a}$ & $0,17 \pm 0,02 \mathrm{~b}$ \\
Diafragma & $0,13 \pm 0,01 \mathrm{a}$ & $0,17 \pm 0,01 \mathrm{a}$ \\
Rins & $0,17 \pm 0,05 \mathrm{a}$ & $0,24 \pm 0,05 \mathrm{a}$ \\
Vísceras verdes & $7,90 \pm 0,45 \mathrm{a}$ & $8,49 \pm 0,44 \mathrm{a}$ \\
Em \% do PV & & \\
& & \\
Carcaça & & \\
Cabeça & $40,50 \pm 0,97 \mathrm{a}$ & $40,81 \pm 0,92 \mathrm{a}$ \\
Patas & $4,02 \pm 0,15 \mathrm{a}$ & $3,86 \pm 0,15 \mathrm{a}$ \\
Pele & $2,16 \pm 0,08 \mathrm{a}$ & $2,15 \pm 0,08 \mathrm{a}$ \\
Coração & $19,75 \pm 0,54 \mathrm{a}$ & $19,34 \pm 0,51 \mathrm{a}$ \\
Pulmões + traquéia & $0,50 \pm 0,03 \mathrm{a}$ & $0,48 \pm 0,03 \mathrm{a}$ \\
Baço & $1,78 \pm 0,06 \mathrm{a}$ & $1,81 \pm 0,05 \mathrm{a}$ \\
Fígado & $0,26 \pm 0,02 \mathrm{a}$ & $0,24 \pm 0,02 \mathrm{a}$ \\
Gordura interna & $1,70 \pm 0,05 \mathrm{a}$ & $1,54 \pm 0,05 \mathrm{~b}$ \\
Diafragma & $0,77 \pm 0,06 \mathrm{a}$ & $0,45 \pm 0,06 \mathrm{~b}$ \\
Rins & $0,39 \pm 0,04 \mathrm{a}$ & $0,45 \pm 0,04 \mathrm{a}$ \\
Vísceras verdes & $0,51 \pm 0,13 \mathrm{a}$ & $0,65 \pm 0,12 \mathrm{a}$ \\
& $23,51 \pm 1,41 \mathrm{a}$ & $22,71 \pm 1,37 \mathrm{a}$ \\
\hline & & \\
& & \\
& & \\
& & \\
& & \\
& &
\end{tabular}

Médias na mesma linha, seguidas de letras diferentes, diferem significativamente entre si $(\mathrm{P}<0,05)$.

Verifica-se, pela Tabela 3, que o peso vivo ao abate foi superior na raça Corriedale, bem como as patas e pulmões + traquéia e estes, porém, somente em valores absolutos, não se observando diferenças na percentagem em relação ao peso vivo ao abate $(\mathrm{P}>0,05)$. Quanto aos percentuais, apenas o fígado na raça Ideal apresentou valores maiores $(\mathrm{P}<0,05)$, não se verificando a mesma tendência quando avaliado em valores absolutos. De acordo com OSÓRIO et al. (1996a), devido ao fato de a influência do genótipo ser determinada pela diferença de maturidade entre as raças, podem-se verificar diferenças entre alguns componentes do peso vivo em valores absolutos e não em valores percentuais, ou vice-versa.

Cabe salientar que a raça Ideal apresentou valores de gordura interna maiores tanto em percentuais do peso vivo como em valores absolutos $(\mathrm{P}<0,05)$. Isso se deve ao fato de ela ser mais precoce que a Corriedale, depositando gordura com menor idade (COSTA et al., 1999).

\section{CONCLUSÕES}

Animais machos castrados, com 1 ano de idade (borregos) da raça Corriedale e Ideal apresentam diferenças morfológicas in vivo e não na carcaça, sendo que os componentes do peso vivo diferem quantitativa e qualitativamente, entre as raças.

\section{REFERÊNCIASBIBLIOGRÁFICAS}

COLOMER, F.; ESPEJO DIAZ, M. Determinación del peso óptimo de sacrificio de los corderos procedentes del cruzamiento Manchego x Rasa Aragonesa en función del sexo. Anales INIA, Serie Producción Animal, n.1, p.103-132, 1971.

COSTA, J.C.C. et al. Estudo da morfologia e características comerciais em cordeiros não castrados de quatro raças. Rev Científica Rural, Bagé, v.4, n.2, p.105-112, 1999.

FRAYSSE, J.L.; DARRE, A. Sur quelles bases économiques et biologiques? Produire des vandes. Paris : Tec. et Doc. - Lavoisier, 1990.

IBGE. SIDRA (Sistema IBGE de Recuperação Automática) Banco de dados agregados. Online. Disponível na Internet http://www.ibge.gov.br/ibge/default.php. Acesso em 22 nov. 2001 .

OLIVEIRA, N.M. et al. Produção de carne em ovinos de cinco genótipos. 5. Estimativas de qualidade e peso de carcaça através do peso vivo. Ciência Rural, Santa Maria, v.28, n.4, p.665669, 1998.

OLIVEIRA, N.M. et al. Morfologia, características comerciais e componentes do peso vivo em cordeiros cruza de Border Leicester com ovelhas Corriedale e Ideal. In: REUNIÃO ANUAL DA SOCIEDADE BRASILEIRA DE ZOOTECNIA, 37, 2000, Viçosa, MG. Anais... Viçosa : SBZ, 2000. p.5. CD Room.

OSÓRIO, J.C.S. et al. Componentes do peso vivo em cordeiros e borregos Polwarth e cruzas Texel x Polwarth. In: CONGRESSO INTERNACIONAL DE ZOOTECNIA, 1991, Evora, Portugal. Anais... Evora : SBZ, 1991. p.49-50.

OSÓRIO, J.C. et al. Componentes do peso vivo em cordeiros e borregos Polwarth e cruzas Texel x Polwarth. Ciência Rural, Santa Maria, v.25, n.1, p.139-143, 1995.

OSÓRIO, J.C. et al. Produção de carne em ovinos de cinco genótipos: 2. Componentes do peso vivo. Ciência Rural, Santa Maria, v.26, n.3, p. 471-475, 1996a.

OSÓRIO, J.C.S. et al. Componentes do peso vivo em cordeiros da raça Corriedale. Ciência Rural, Santa Maria, v.26, n.3, p.483-487, 1996 b.

OSÓRIO, J.C.S. et al. Produção de carne em ovinos de cinco genótipos. 3. Perdas e morfologia. Ciência Rural, Santa Maria, v.26, n.3, p.477-481, 1996 c.

OSÓRIO, J.C.S. et al. Métodos para avaliação de produção de carne ovina: in vivo, na carcaça e na carne. Pelotas, RS: Universidade Federal de Pelotas, Departamento de Zootecnia, 1998. 107p.

OSÓRIO, J.C.S. et al. Efecto de la edad al sacrificio sobre la producción de carne en corderos no castrados de cuatro razas. Rev Bras de Agrociência, Pelotas, v.6, n.2, p.161-166, 2000 . 
PRADAL, M. Produire de la viande bovine aujourd'hui Paris : Tec. et Doc. - Lavoisier, 1989.

SAÑUDO, C.; SIERRA, I. Calidad de la canal en la especie ovina. Barcelona, España : One, 1986. p.127-153.

SAS. User's Guide: Statistics. Cary, NC, 1982. 584p.
SIERRA, I.; OSÓRIO, M.T.; OSÓRIO, J.C.S. Producción de corderos ligeros en la raza Rasa Aragonesa, Ojinegra de Teruel Y Roya Bilbilitana. I. Calidad de la canal. In: JORNADAS CIENTÍFICAS DE LA SOCIEDAD ESPAÑOLA DE OVINOTECNIA Y CAPRINOTECNIA, 18, 1993, Albacete, España. Anais... Albacete : SEOC, 1993. 